\title{
What Java Developers Know About Compatibility, And Why This Matters
}

\author{
Jens Dietrich \\ School of Engineering and Advanced Technology \\ Massey University, Palmerston North, New Zealand \\ email:j.b.dietrich@massey.ac.nz \\ Kamil Jezek, Premek Brada \\ Faculty of Applied Sciences \\ University of West Bohemia, Pilsen, Czech Republic \\ \{kjezek, brada\}@ntis.zcu.cz
}

August 13, 2014

\begin{abstract}
Real-world programs are neither monolithic nor static - they are constructed using platform and third party libraries, and both programs and libraries continuously evolve in response to change pressure. In case of the Java language, rules defined in the Java Language and Java Virtual Machine Specifications define when library evolution is safe. These rules distinguish between three types of compatibility - binary, source and behavioural. We claim that some of these rules are counter intuitive and not well-understood by many developers. We present the results of a survey where we quizzed developers about their understanding of the various types of compatibility. 414 developers responded to our survey. We find that while most programmers are familiar with the rules of source compatibility, they generally lack knowledge about the rules of binary and behavioural compatibility. This can be problematic when organisations switch from integration builds to technologies that require dynamic linking, such as OSGi. We have assessed the gravity of the problem by studying how often linkage-related problems are referenced in issue tracking systems, and find that they are common.
\end{abstract}

\section{Introduction}

Modern programming languages like Java support dynamic linking where a client program can be executed with libraries that have been compiled separately. While the libraries used at runtime are usually also present when the program is compiled, it is not required that the same versions of these libraries 
are used for compilation. This addresses some important use cases, such as the deployment of newer versions of a library with improvements such as bug fixes or better performance. As long as the APIs (application programming interfaces) defined in these libraries don't change, this works well. Unfortunately, APIs do change when libraries evolve $[13,8,12]$. When this happens, programmers are suddenly confronted with different sets of rules [9]: the rules of source compatibility are used by the compiler when a program is compiled against a library, while the rules of binary compatibility are used when a program is linked against a library that has been compiled separately. To make things even more complicated, the Java Language Specification defines binary compatibility strictly with respect to linking [15, ch. 13]. This does not cover all problems that can occur when the respective program is executed with a particular library in the classpath. This leads to a third type of compatibility, behavioural compatibility.

To illustrate the different types of compatibility, consider the following examples in listing 1 and 2. Both examples consist of a class Main that is compiled with the first version of a class Foo in a library $1 \mathrm{ib}-1.0 . j \mathrm{ar}$, and then executed with another version of Foo in a library lib-2.0.jar.

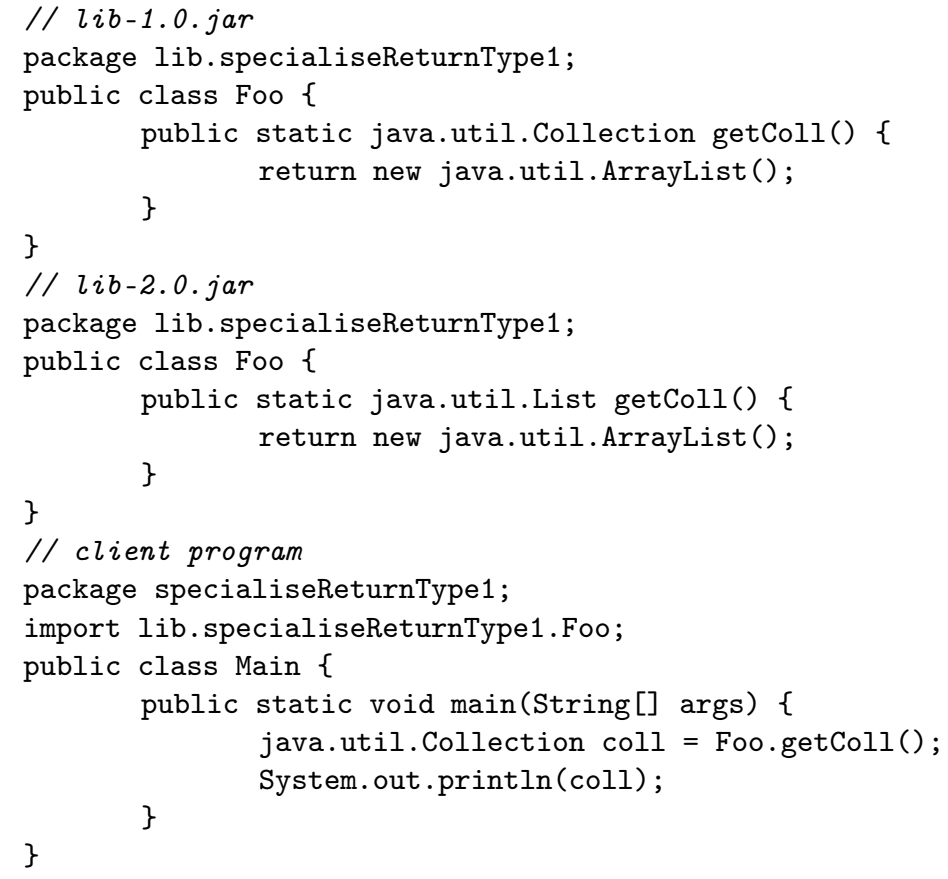

Listing 1: Specialising the return type of a method

These two examples demonstrate that the different types of compatibility are inconsistent and not intuitive. In the first example, the return type of a used method is specialised. While this is usually source compatible ${ }^{1}$, it is not

${ }^{1}$ The exception is when the method is overridden 
binary compatible as the linker does not use subtype reasoning when a method reference is resolved. In this case, a NoSuchMethodError is thrown. In other words, the only required correction is recompilation of the program with version 2.0 of the library. No source code modification is needed.

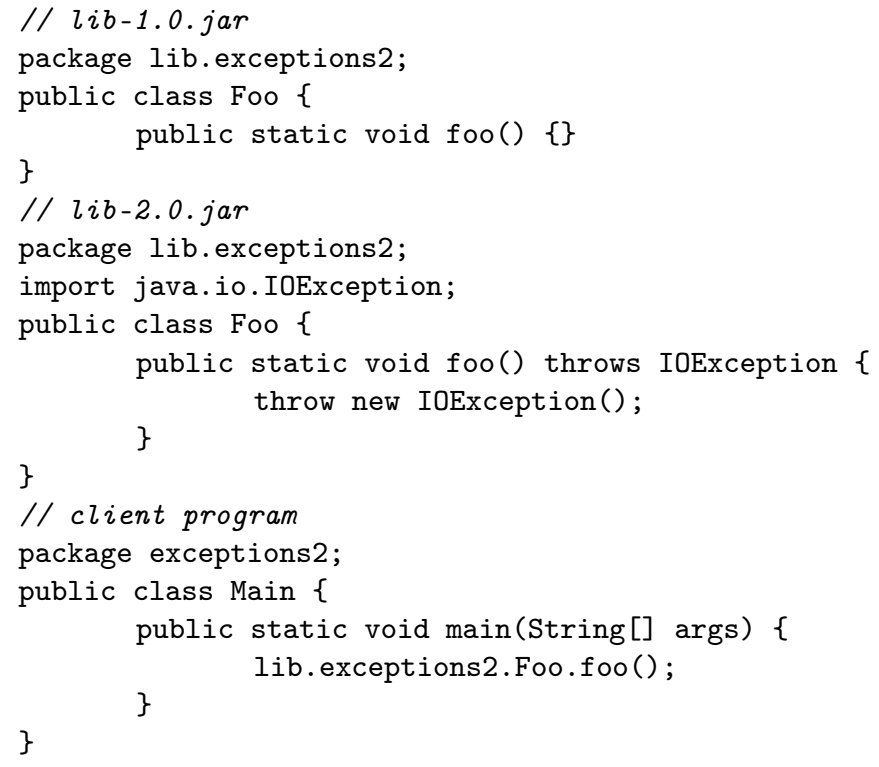

Listing 2: Adding a checked exception to a method

The second example shows that while information about the exceptions thrown is present in the byte code as part of the signature [20, ch. 4.3.4], and can even be queried through the reflection API, this information is not part of the descriptor [20, ch. 4.3.3] used for linking. I.e., the uncaught checked exception is not detected through static analysis during linking, but only at runtime when the exception is actually thrown when $f \circ o()$ is invoked, and the program exits with an error. This is therefore an example of behavioural incompatibility, although it would be more intuitive if this was binary incompatible.

The mismatch between the different notions of compatibility has increased as the Java language has evolved. The development of Java language was driven by programmer productivity, while preserving binary compatibility [10]. Features like nested and inner classes, generic types and auto boxing / unboxing have added simplicity and expressiveness to the language with minimal or no changes to the byte code format. In case of generic types, this has lead to erasure. But even changing a field type from a primitive type to its wrapper type or vice versa breaks binary compatibility (listing 3 ), even though this problem can easily be solved by recompilation as the compiler applies auto boxing or unboxing, respectively.

// $l i b-1.0 . j a r$

package lib.primwrap1; 


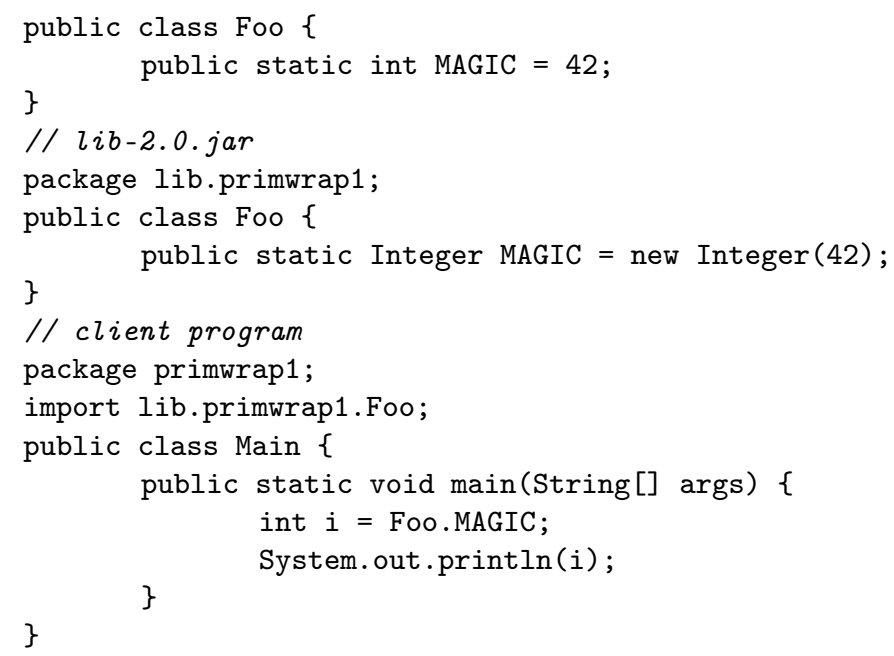

Listing 3: Wrapping a primitive field

It is reasonable to ask here why this matters. In many cases, a project is built (compiled and unit tested) against the very libraries it uses at runtime, and widely used build and continuous integration tools facilitate this approach. This circumvents the problem. However, if libraries are individually upgraded, problems start to occur. The JLS envisages this approach: "Development tools for the Java programming language should support automatic recompilation as necessary whenever source code is available. Particular implementations may also store the source and binary of types in a versioning database and implement a ClassLoader that uses integrity mechanisms of the database to prevent linkage errors by providing binary-compatible versions of types to clients" [15, ch. 13]. While preserving binary compatibility is a major objective for the JRE [10], this is not the case for many other libraries. In our previous work [12] we have shown that binary compatibility is often broken when open source libraries evolve. In particular, this applies to popular libraries such as ANT, ANTLR, Hibernate and Lucene. The major reason that this matter is, however, the popularity of OSGi [25] - a framework that heavily relies on dynamic linking to support a flexible lifecycle for components in long-running, high-availability applications. The problem might be further accelerated when modularity support is added to the Java platform (project Jigsaw), although it is not clear at this point whether Jigsaw will support dynamic modules [4].

The rest of this paper is organised as follows. In section 2, we discuss the design of the survey we conducted to find out what developers know about the different types of compatibility. We analyse the responses in section 3. In section 4 , we try to assess the size of the problem in practice by analysing issue tracking systems for references to errors caused by linkage related problems. In section 5 we discuss several treats to validity. We finish the paper with a conclusion, including a discussion of related and future work. 


\section{Survey Design}

The survey is based on a set of Java library evolution puzzlers we have developed for training ${ }^{2}$, in a style inspired by [5]. The full survey contains 7 questions about the background of the respondent, and 25 puzzlers - 21 standard puzzlers with 2 questions each, and 4 constant inlining puzzlers with 1 question per puzzler - a total of 46 technical questions. A few days after the survey had opened we realised that many respondents only answered the first few questions, and then abandoned the survey. We therefore created a second, shorter survey with the same set of 7 background questions, 9 standard and 4 constant inlining puzzlers, a total of 22 technical questions. The question in the short survey are a subset of the questions in the full survey.

\subsection{Respondent Background Questions}

We have asked respondents the following set of background questions to assess their level of relevant experience.

1. Which programming languages do you regularly use? Choices were: Java, C, C++, Python, Ruby, ObjectiveC, C\#, JavaScript, other JVM-based languages (Scala, Groovy, etc), other.

2. How many years of Java programming experience do you have? Choices were: less than 1 year, 1-3 years, 4-10 years, more than 10 years.

3. How would you rank your Java expertise? Choices were: novice, some experience, knowledgeable, expert, guru.

4. Are you familiar with the following Java concepts? Only answer this question if you chose novice as an answer to the previous question. Choices were: interfaces, inheritance, the difference between source code and byte code, the difference between errors, checked and runtime exceptions, wrapper types (boxing/unboxing), generic parameter types, the classpath.

5. Which of the following technologies have you used? Choices were: Spring, OSGi, OSGi extensions (Eclipse plugins, Spring DM, etc), WebStart, J2EE application servers, ASM, BCEL or other byte code analysis tools, AspectJ or other AOP tools, Antlr or other parser generators, Ant, Maven or other build tools, Jenkins, Hudson or other continuous integration tools.

6. Have you designed frameworks, libraries or APIs? This was a yes/no question.

7. What is your main occupation? Choices were: student, academic, industry research, programmer, project manager.

\footnotetext{
${ }^{2}$ http://www.slideshare.net/JensDietrich/presentation-30367644
} 


\subsection{Technical Questions Overview}

The technical questions are summarised in table 1. Each question in the table belongs to a category and the question's puzzler is implemented as a simple program. Puzzlers are split into packages, the respective package names are shown in the third table column. Given a package name <pck>, each puzzler consists of three classes named as follows:

1. a class lib. $\langle$ pck $>$.Foo packaged in a library $1 \mathrm{ib}-1.0 . j \mathrm{jar}$

2. a (modified) class lib. $\langle$ pck $>$.Foo packaged in a library lib-2.0.jar

3. a class $\langle$ pck $>$. Main with a main method that uses $l i b .<p c k>$. Foo

The code and an ANT script to execute the experiments is available from the following public code repository:

https://bitbucket.org/jensdietrich/java-library-evolution-puzzlers

The source code for the questions is in the /examples folder and its respective sub folders for both versions of the library and the client program. The root folder contains an ANT script that can be used to compile and execute the respective scenario. The correct answers are defined with respect to the output of this script, confirmed by cross-referencing the output with the Java Language Specification [15]. Given a unique package name $\langle$ pck $>$, the following command can then be used to run the compilation and linking script for this puzzler / question:

ant -Dpackage $=$ pck

There are two types of problems. All problems except the problems in the constant inlining category are standard puzzlers. For each standard puzzler, we have asked the following two questions:

1. Can the version of the client program compiled with lib-1.0.jar be executed with lib-2.0.jar? The three possible answers are:

(a) no, an error occurs

(b) yes, but the behaviour of the program changes

(c) yes, and the behaviour of the program does not change

2. Can the client program be compiled and then executed with $1 \mathrm{ib}-2.0 \cdot \mathrm{jar}$ ? The three possible answers are:

(a) no, compilation fails

(b) yes, but the behaviour of the program is different from the program version compiled and executed with $1 \mathrm{ib}-1.0 . \mathrm{jar}$ 
(c) yes, and the behaviour of the program is the same as the program version compiled and executed with lib-1.0.jar

Note that we avoided references to the standard definitions of binary and source compatibility in the specification documents on purpose, as we can not assume that the majority of developers is familiar with these documents. We defined a behaviour change as follows in the survey: "a behaviour change is either a different console output or a situation where the execution of one program version throws an exception, but the the execution of the other program version does not".

Table 1 uses wildcards in the question ids. The wildcard is replaced by "UPGR" (dynamic upgrade) for the first question, and by "-RECP" (recompile and upgrade) for the second question. For the constant inlining category, only one question is asked. This will be explained below. We refer to these three question types as UPGR, RECP and INL, respectively.

\section{$2.3 \quad$ Interface Puzzlers}

The puzzlers in this category describe problems that occur when methods are added to or removed from interfaces implemented by a client program. In IF1, a method is added to the interface that is not actually being used by the class implementing the interface. This is binary compatible, but not source compatible. To solve the other problems in this category, respondents also had to understand the @Override annotation (IF2, IF3) and Java method lookup (IF4).

\subsection{Method Descriptor Puzzlers}

The descriptor of a method is the combination of parameters plus the return type, and this information is used at runtime for linking and method dispatch [15, ch. 15.12.2]. When refactoring methods, specialising return types and generalising parameter types is generally safe. This can be seen as special cases of strengthening post-conditions and weakening pre-conditions, respectively.

However, in both cases the method descriptors are changed, causing binary incompatibility. More precisely, this results in an instance of NoSuchMethodError being thrown. SP_RET1 is shown in listing 1 . The other questions in this category are variants of this question, also including the narrowing of primitive return types (SP_RET2) and the (unsafe) widening of primitive parameter types (GEN_PAR3). GEN_PAR2 is a scenario where generalising a parameter type leads to ambiguity and compilation fails.

\subsection{Exception Puzzlers}

Exceptions (including checked exceptions) are not part of the descriptor. This means that changes to the exceptions declared by a method like adding or generalising are generally binary compatible but often behavioural incompatible. 
Table 1: Question Overview

\begin{tabular}{|llll|}
\hline category & question id & package name & $\begin{array}{l}\text { short sur- } \\
\text { vey }\end{array}$ \\
\hline \hline interfaces & IF1-* & addtointerface & yes \\
interfaces & IF2-* & removefrominterface1 & no \\
interfaces & IF3-* & removefrominterface2 & no \\
interfaces & IF4-* & removefrominterface3 & no \\
\hline method descriptors & SP_RET1-* & specialiseReturnType1 yes \\
method descriptors & SP_RET2-* & specialiseReturnType2 no \\
method descriptors & SP_RET3-* & specialiseReturnType3 no \\
method descriptors & SP_RET4-* & specialiseReturnType4 no \\
method descriptors & GEN_PAR1-* & generaliseParamType1 yes \\
method descriptors & GEN_PAR2-* & generaliseParamType2 no \\
method descriptors & GEN_PAR3-* & generaliseParamType3 yes \\
\hline exceptions & ADD_EXC1-* & exceptions1 & no \\
exceptions & ADD_EXC2-* & exceptions2 & yes \\
exceptions & SPEC_EXC1- & exceptions4 & no \\
& $*$ & & \\
exceptions & REM_EXC1- & exceptions5 & yes \\
& $*$ & & \\
exceptions & REM_EXC2- & exceptions6 & no \\
& $*$ & & \\
\hline auto (un)boxing & BOX1-* & primwrap1 & yes \\
auto (un)boxing & BOX2-* & primwrap2 & no \\
\hline generic param types & GEN1-* & generics1 & yes \\
generic param types & GEN2-* & generics2 & yes \\
\hline constant inlining & CON1 & constants1 & yes \\
constant inlining & CON2 & constants2 & yes \\
constant inlining & CON3 & constants3 & yes \\
constant inlining & CON4 & constants4 & yes \\
\hline others & NEST-* & ghost & no \\
\hline
\end{tabular}

This is the case in example ADD_EXC2 (listing 2). ADD_EXC1 is a similar scenario using a runtime exception. On the other hand, certain changes to declared exceptions that seem uncritical like removing a declared exception can lead to source incompatibilty as the compiler detects that catch blocks become unreachable [15, ch. 14.21]. Question REM_EXC1 is based on such a scenario. Question REM_EXC2 is noteworthy as the behaviour of the current Java (OpenJDK SE Runtime Environment 1.7.0_45-b18) implementation differs from the specification due to a bug in the JLS. This bug was reported and accepted $^{3}$, and we defined the correct answer for this question with respect to the behaviour of the Java 7 implementation used.

${ }^{3}$ Email conversation with Alex Buckley, 11 October 2013 


\subsection{Auto (Un)Boxing Puzzlers}

Auto boxing / unboxing were introduced in Java 5.0. This feature hides the differences between object (wrapper) types and their corresponding primitive types. However, on the byte code level they are still treated as completely different types. In BOX1 (listing 3), the library declares a public field of type int that is read by the client to assign a value to a variable declared as int. The type of this field is then changed to java.lang. Integer. This is not binary compatible but source compatible as the compiler can apply auto unboxing. More precisely, this results in an instance of NoSuchFieldError to be thrown. BOX2 reverses this scenario - the field type is changed from java. lang. Integer to int. The result is the same.

\subsection{Generic Type Puzzlers}

Generic types were also introduced in Java 5.0. Erasure [15, ch. 4.6] is used to achieve binary compatibility so that existing non-generic clients can use generic code. The questions in this category describe two situations resulting from this. In the first puzzler (GEN1) the library defines a method List<String> getList () that returns a list containing " 42 ". This method is then changed to a method List<Integer> getList() that returns a list containing 42 . The client accesses the list, assigns it to a variable List<String $>$ list and prints its size to the console. Due to erasure, this is binary compatible, but not source compatible as the compiler detects that the assignment is illegal. The second puzzler (GEN2) is very similar, but this time the client loops over the list and prints its element to the console. This is still binary compatible but fails with a class cast exception due to the checkcast statements generated by the compiler when the elements of the list are accessed. This is therefore an example of behavioural incompatibility.

\subsection{Constant Inlining Puzzlers}

The structure of the four questions in this category is slightly different. Listing 4 shows the code used in CON1.

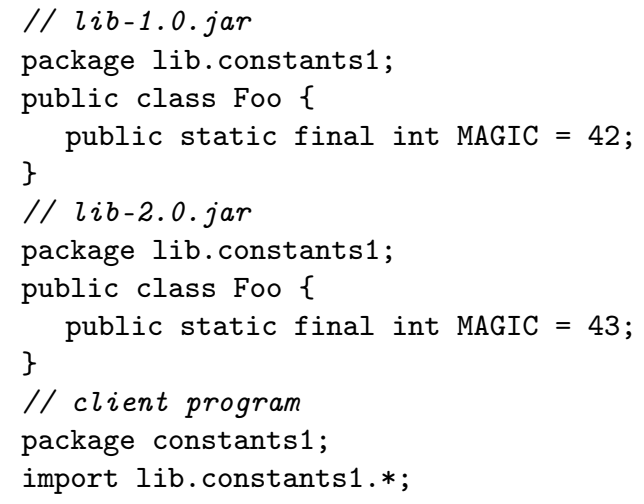




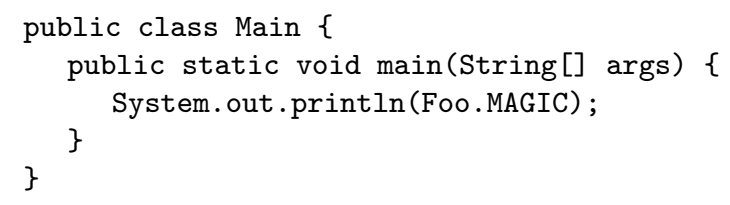

Listing 4: Constant Inlining

There is only one question for each problem in this group: whether 42 or 43 is printed to the console when the program compiled with version 1.0 of the library is executed with version 2.0 of this library. CON2 uses strings instead of integers, CON3 defines an integer constant using an expression instead of a literal, and in CON4 the wrapper type java.lang. Integer is used instead of int. It turns out that constants of type int and String are inlined, and that the compiler uses constant folding to evaluate expressions. This means that 42 is printed when the programs in $\mathrm{CON} 1, \mathrm{CON} 2$ or CON3 are executed. However, Integer values are not inlined, and 43 is printed in case of CON4.

\subsection{Other Puzzlers}

NEST is a scenario where the client program uses a static method defined in a class lib.ghost.Foo.Bar. In the first version, Bar is a static nested class within lib.ghost.Foo, while in the second version Bar is refactored to a top-level class within the package lib.ghost.Foo. This is binary incompatible but source compatible as the byte code represention changes from lib/ghost/Foo $\$ B a r$ to lib/ghost/Foo/Bar while the source code representation (lib.ghost.Foo.Bar) remains the same.

\section{Responses}

\subsection{Access to Raw Survey Data}

The raw data exported from SurveyMonkey can be accessed in the following repository:

https://bitbucket.org/jensdietrich/java-library-evolution-puzzlers

The data is available as a set of Excel files in the /survey/results/rawdata/ folder.

\subsection{Overview}

The survey was open between 15 November and 31 December 2013. During this period, 184 respondents started the short version of the survey, while 241 respondents started the full version. We asked respondents doing the full survey whether they had already completed the short survey. Only 11 respondents answered yes. Assuming that everybody answered this question correctly, and 
nobody completed the short survey after the full survey, this gave us 414 unique respondents. To avoid double counting, we removed all answers for questions that are in both surveys for responses by people who indicated that they had completed both surveys.

The number of valid answers for the technical questions ranged from 49 (REM_EXC2-RECP) to 295 (IF1-UPGR and IF1-RECP).

\subsection{Background of Respondents}

The vast majority of respondents are programmers (figure 1), have 4 or more years of experience with Java technologies (figure 3) and self-assess their familiarity with Java technology as either knowledgeable or expert (figure 2).

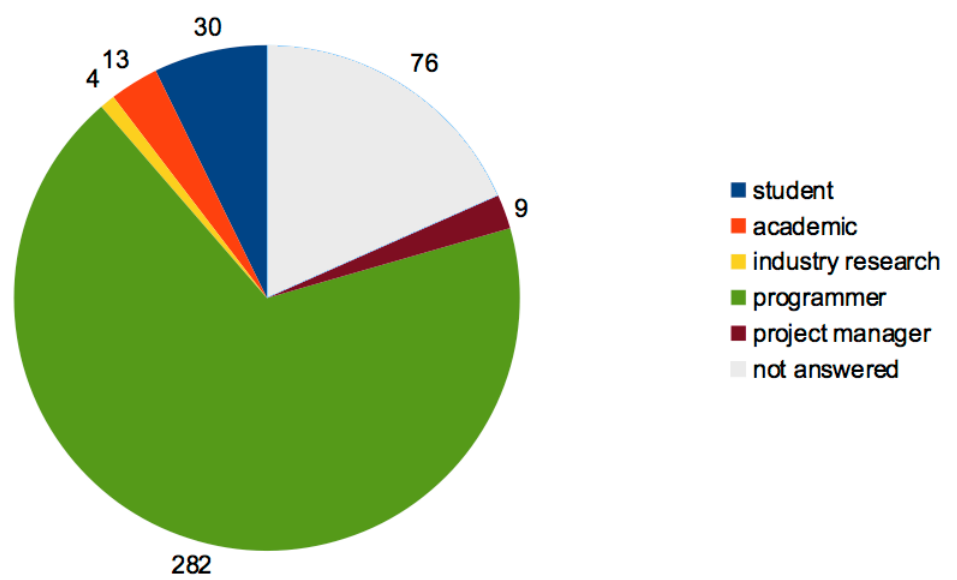

Figure 1: Occupation of survey respondents

\subsection{Analysis}

Of all the answers provided by respondents, only $62 \%$ were correct. The percentage of correct answers is much better for the RECP questions (76\%) than for the UPGR (51\%) and INL (52\%) questions. This indicates that respondents are more familiar with the rules of source compatibility than with the rules of binary and behavioural compatibility.

Figures 4, 5 and 6 show the total numbers of correct and incorrect answers for the questions in the UPGR, RECP and INL categories, respectively. The spikes in the total number of responses represent questions that were part of the short survey. In general, there are more wrong answers for the questions that were also part of the short survey. This is due to the fact that more experienced developers completed the full survey.

The correct answer ratio is particularly low for the first motivational example discussed in the introduction: only $27 \%$ of respondents answered SP_RET1- 


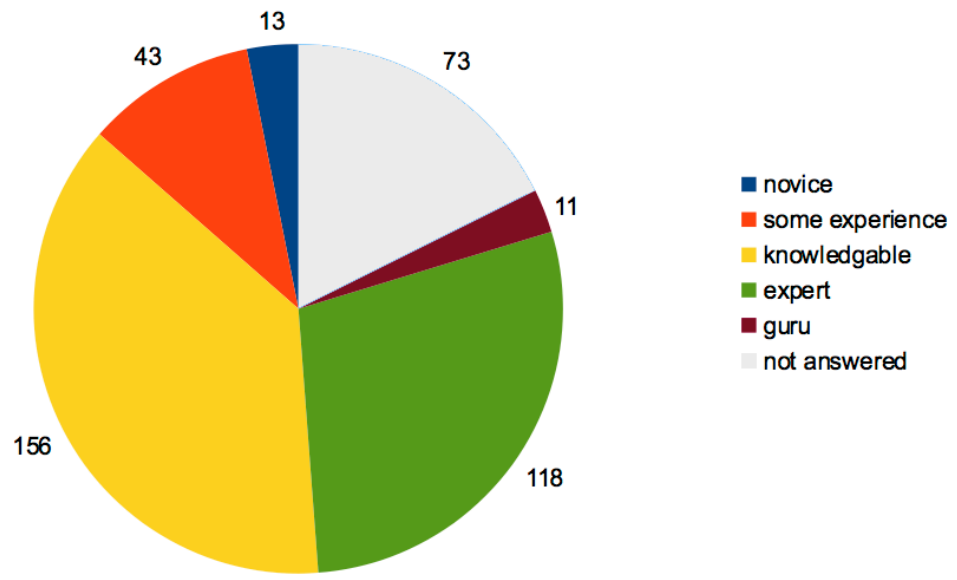

Figure 2: Self-assessed level of Java experience of survey respondents
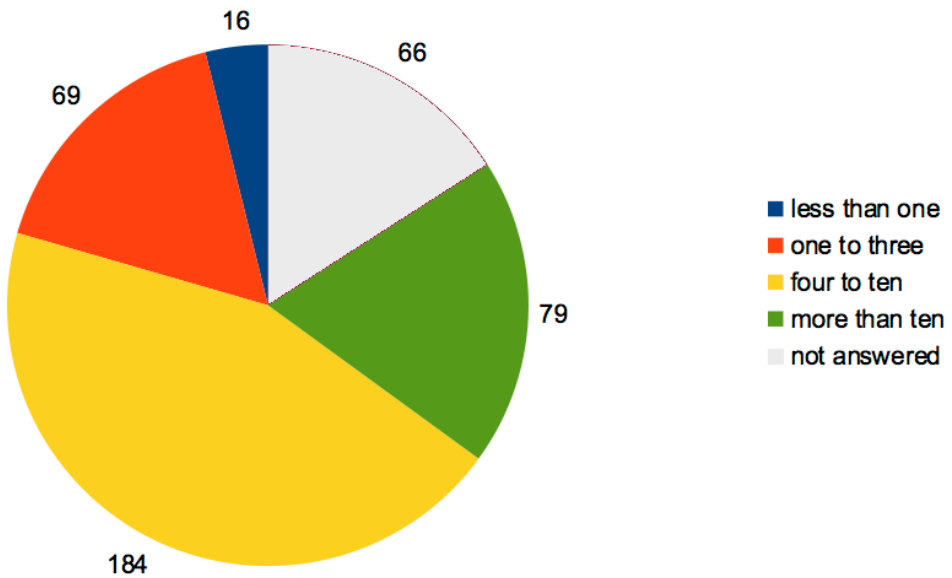

Figure 3: Years of Java experience of survey respondents

UPGR (listings 1) correctly. Other questions with a high percentage of wrong answers are GEN_PAR1-UPGR (27\% correct, generalising a parameter type is binary incompatible), and GEN2-UPGR (20\%). In the case of GEN2-UPGR (see also section 2.7), many respondents answered that the upgraded library is binary incompatible. However, it is only behavioural incompatible - no linkage error is generated, but a runtime exception (ClassCastException) is thrown when the program is executed.

While most respondents answered the RECP type questions correctly, only $30 \%$ got GEN_PAR3-RECP (listing 5) right. This question is inspired by the classical Java puzzlers [5], where compilation succeeds but the behaviour of the 


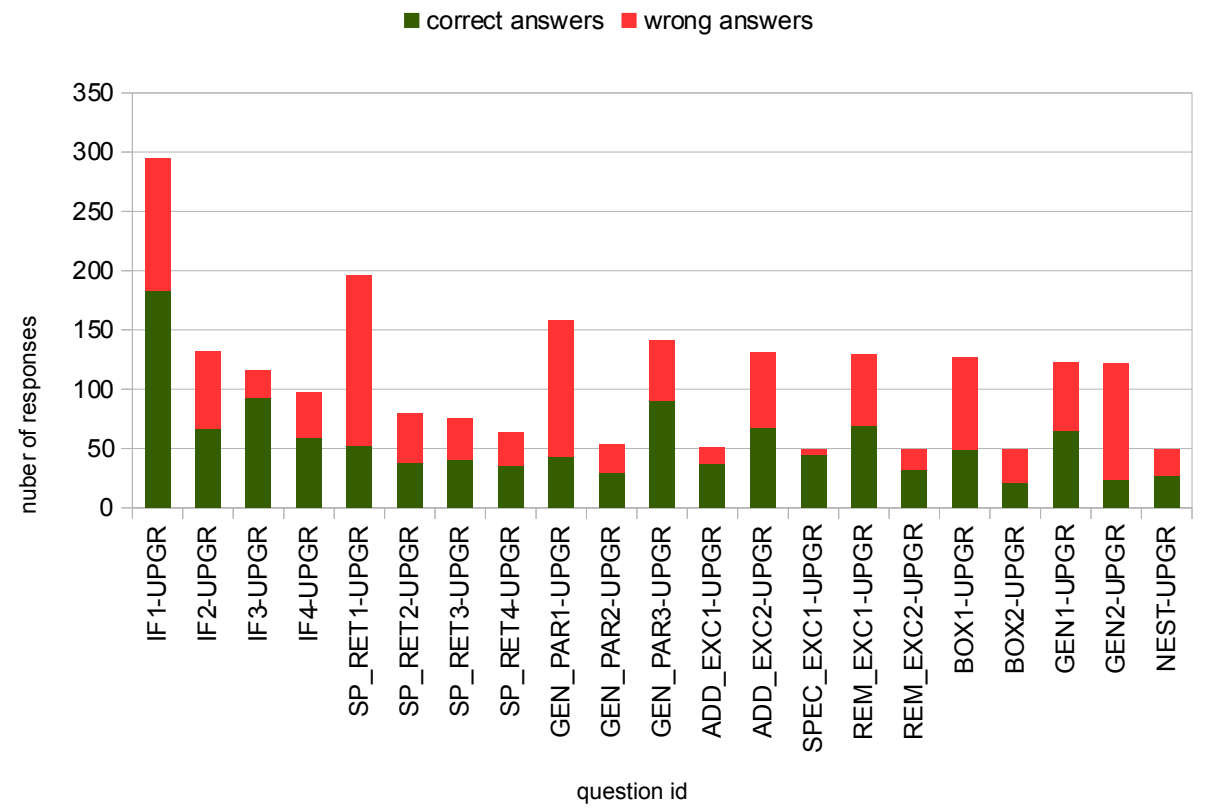

Figure 4: Overview - answers for UPGR (library upgrade) questions

program is changed by the recompilation due to the loss of precision when the widening conversion from int to float is performed [15, ch. 5.1.2].

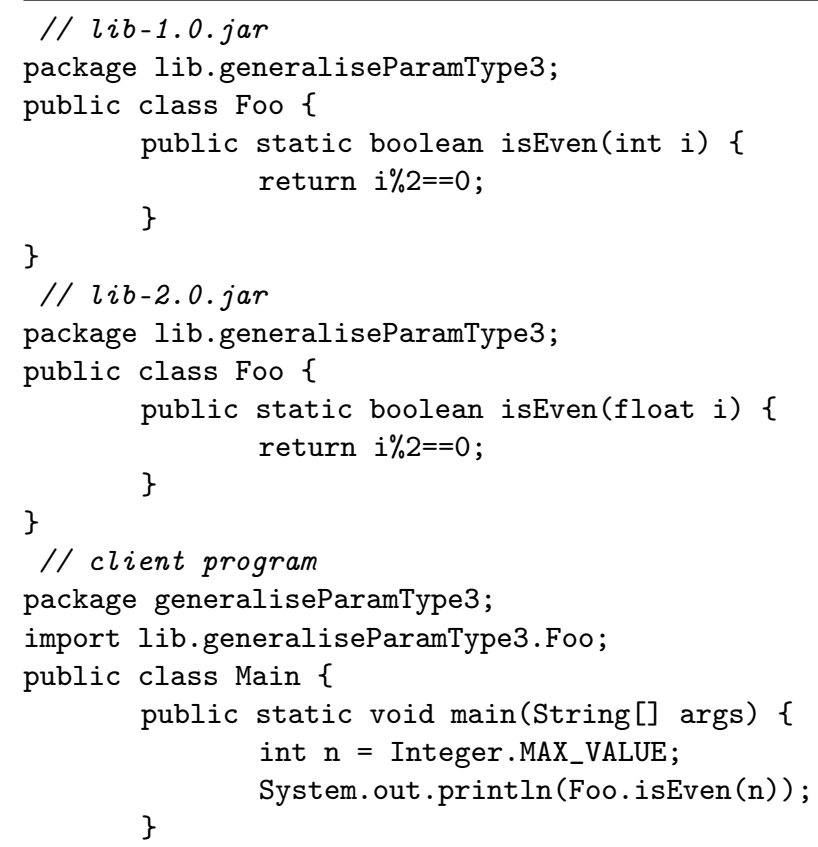




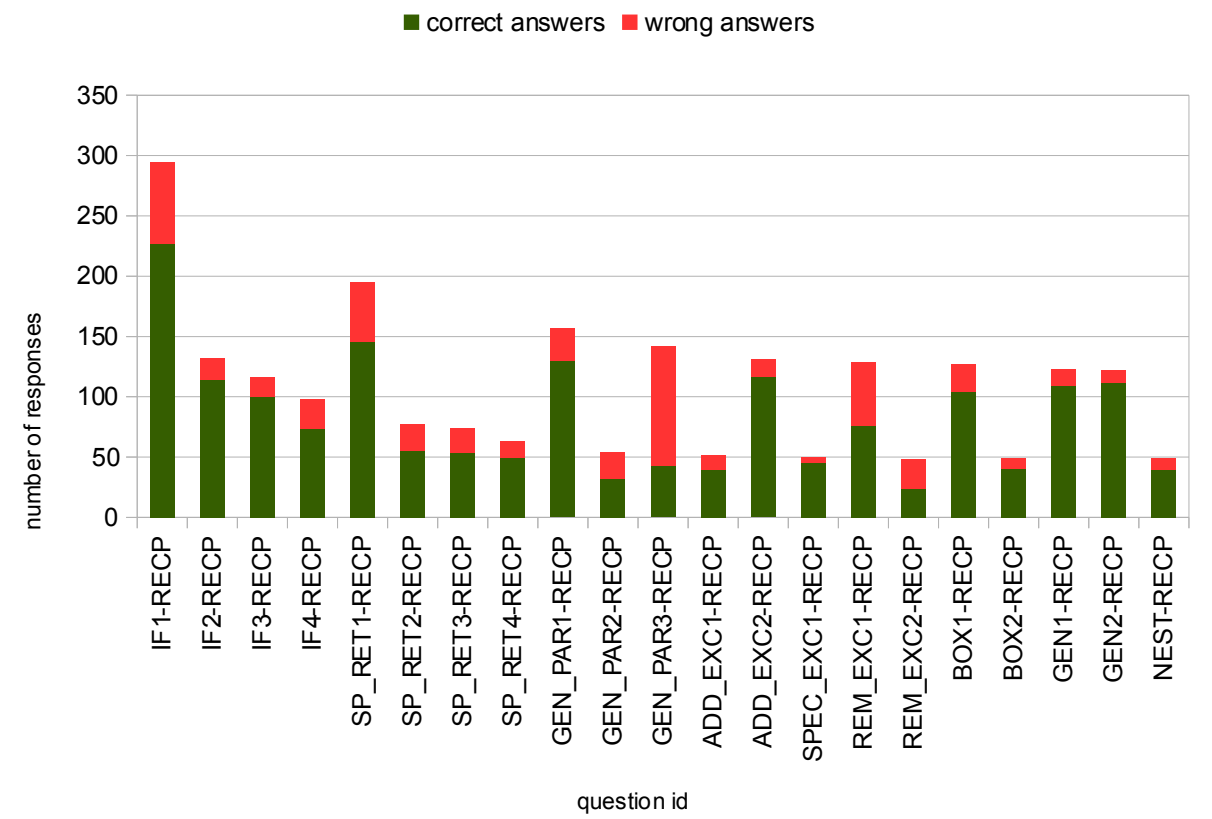

Figure 5: Overview - answers for RECP (recompilation) questions

\}

Listing 5: Generalising a Parameter Type

Constant inlining is not well understood either, only $53 \%$ of respondents answered the most simple question (CON1), inlining of a constant defined by an int literal, correctly. This number drops further to $33 \%$ for inlining of string type constants (CON2), and $46 \%$ for inlining of constants defined by expressions (inlining through constant folding, CON3). The relatively high number of correct answers for CON4 is probably misleading. Constants declared using wrapper types (Integer in this case) are not inlined, and we assume that many respondents answered this question accidentally correctly as they were not aware of the concept on inlining in the first place.

Figures 7 and 8 show the dependency of correct answers on years and level of experience with Java. Not surprisingly, answers improve with increasing experience, but not to the extent we had expected. Even (self-assessed) expert / guru users and users with more than 10 years experience of Java technology answered only $60 \%$ of the questions in the UPGR category correctly. Surprisingly, the number of correct answers from participants with less than one year experience is slightly higher than the respective number for participants with 1-3 years experience. We think that this is caused by a few undergraduate students taught by the authors participating in the study. This particular cohort has some basic understanding of some of the issues involved. 


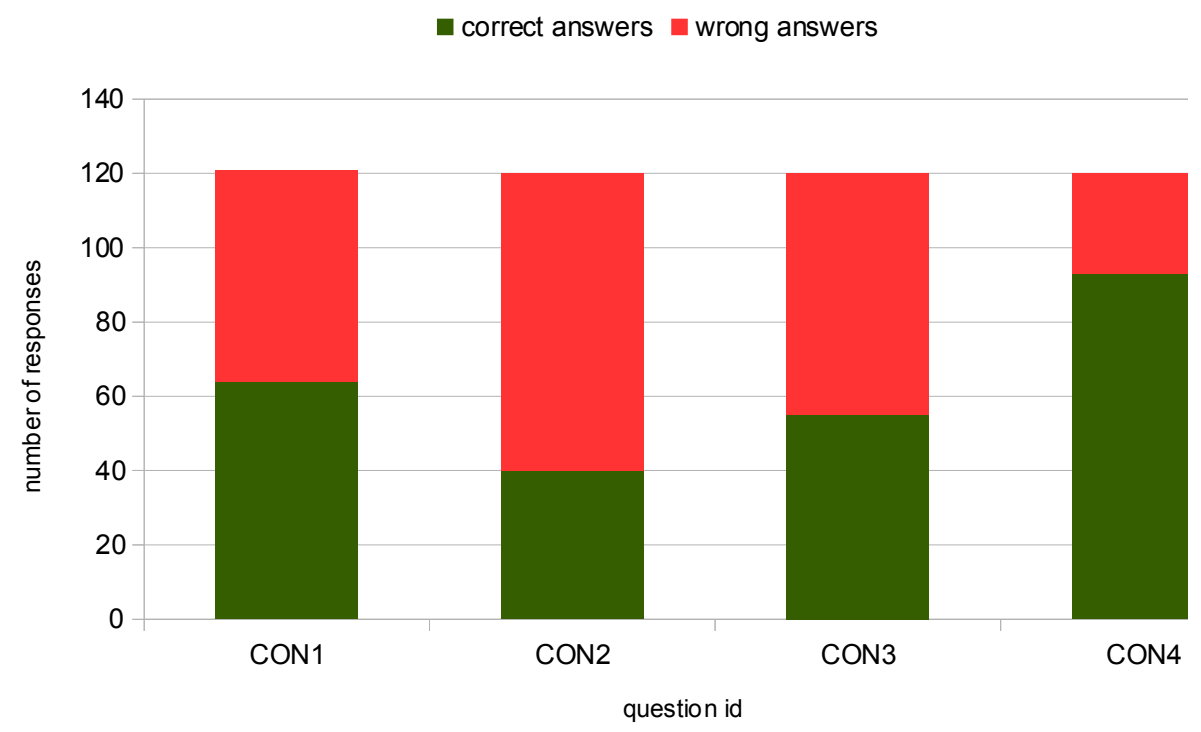

Figure 6: Overview - answers for constant inlining puzzler questions

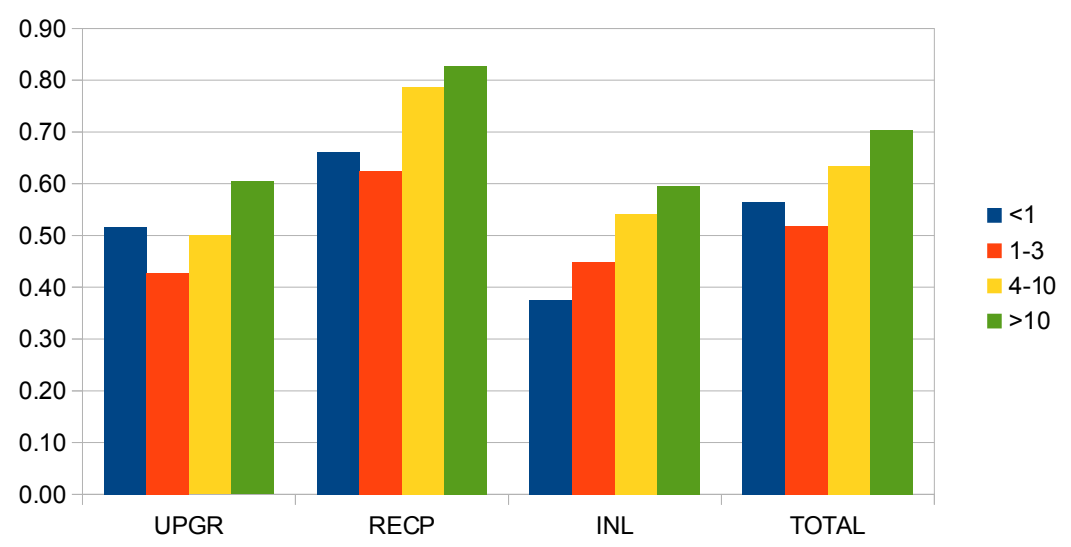

Figure 7: Ratio of correct answers by years of java experience

We further tried to work out the notion of an expert user by combining answers to the several contextual questions in the beginning of the survey. For this purpose, we defined a pro respondent profile: a respondent with framework design experience, an expert or guru level of experience, at least four years of experience with Java technology and knowledge in at least one additional programming language. As OSGi is the "killer technology" for dynamic library upgrades, we also defined a second pro+osgi profile based on the same criteria as pro plus the requirement that the respondent must have OSGi experience. For 


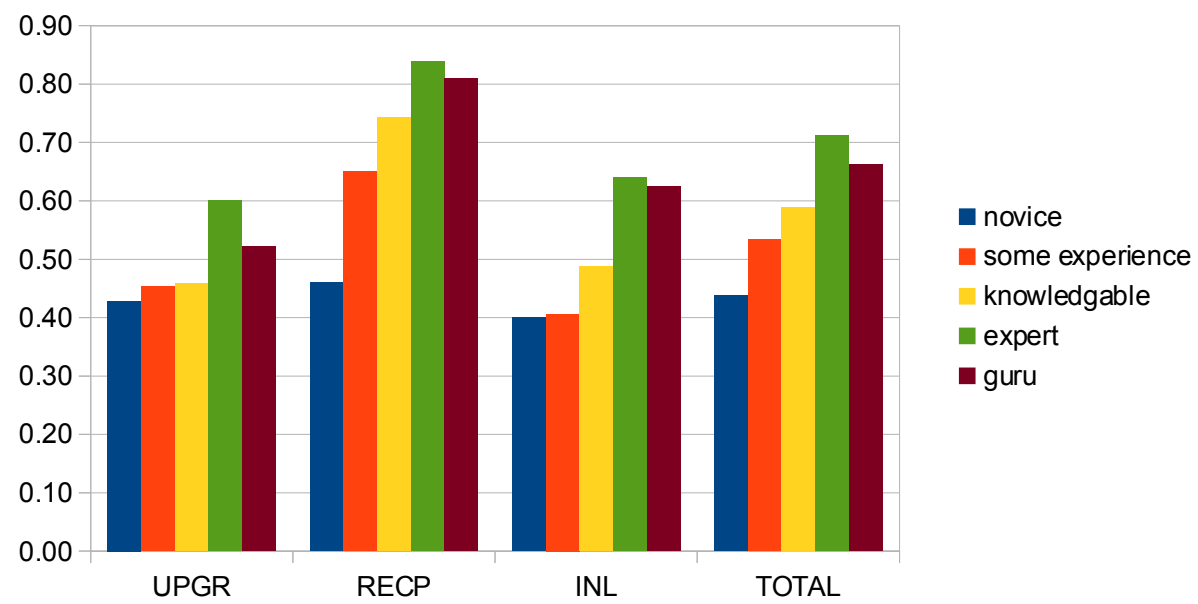

Figure 8: Ratio of correct answers by self-assessed level of experience

both profiles, we still had reasonable numbers of respondents and answers. The respective results are summarised in table 2 . Not surprising, pro and pro + osgi respondents answered more questions correctly. pro + osgi respondents do significantly better for the questions in the UPGR category - they clearly better understand binary compatibility. However, overall the results in this category are still lower than expected.

Table 2: Question Overview

\begin{tabular}{|l|l|l|l|l|}
\hline questions & & all & pro & pro + osgi \\
\hline \hline \multirow{4}{*}{ all } & correctness & $63 \%$ & $74 \%$ & $78 \%$ \\
& answer count & 5006 & 680 & 474 \\
& respondent count & 414 & 38 & 23 \\
\hline \multirow{3}{*}{ UPGR } & correctness & $51 \%$ & $62 \%$ & $69 \%$ \\
& answer count & 2270 & 308 & 217 \\
& respondent count & 414 & 38 & 23 \\
\hline \multirow{3}{*}{ RECP } & correctness & $76 \%$ & $88 \%$ & $89 \%$ \\
& answer count & 2263 & 308 & 217 \\
& respondent count & 414 & 38 & 23 \\
\hline \multirow{3}{*}{ INL } & correctness & $53 \%$ & $67 \%$ & $70 \%$ \\
& answer count & 473 & 64 & 40 \\
& respondent count & 414 & 38 & 23 \\
\hline
\end{tabular}




\section{Impact Analysis}

\subsection{Introduction}

While the survey results demonstrate that many developers have only limited knowledge about the different kinds of compatibility rules in Java, it is not clear what impact this has on the quality of systems and developer productivity. In particular, binary compatibility matters when systems use partial upgrades. This is the case for OSGi-based technology that is used in many developer tools and in major application servers. We therefore suspect that the lack of knowledge on compatibility has an impact on both the quality of products and the productivity of developers. In our previous work, we found some evidence that compatibility-related problems are common in real-world systems, and can break compatibility if libraries are updated [12].

\subsection{Methodology}

To investigate the gravity of the problems caused by the lack of knowledge about compatibility rules, we focused on binary compatibility. The reason is that binary compatibility has a very precise definition in the Java Language Specification [15, ch. 13], and violating its rules results in certain types of linkage errors. These errors instantiate subclasses of java.lang.LinkageError. This makes it easy to search for these problems in issue tracking systems as many developers copy and paste stack traces and error messages containing the fully qualified class name of the respective error class.

It is difficult to interpret absolute numbers, i.e., the number of reports for a particular error, correctly. We therefore decided to assess the number of linkage errors reported relative to the number of reported errors and exceptions widely considered as common. We selected two (non linkage) errors and two runtime exceptions for the baseline ${ }^{4}$ :

1. NullpointerException - this is probably the most frequently encountered exception in Java, thrown when an application attempts to use null in a case where an object is required.

2. ClassCastException - thrown to indicate that an application has tried to cast an object to a subclass of which it is not an instance.

3. StackOverflowError - thrown if an application recurses too deeply, usually the result of an erroneous termination condition.

4. OutOfMemoryError - thrown if the application runs out of heap space, e.g. if too many objects have been allocated and cannot be garbage collected.

By using these four classes as a base line, we assume that these errors and exceptions all represent problems that are frequently encountered by developers.

\footnotetext{
${ }^{4}$ All error and exception classes referenced in this section are defined in the java.lang package.
} 
In most cases, they result from mistakes made by programmers, as opposed to integration problems caused by the configuration of classpath settings or build scripts. We have compared the frequency of problems with references to these errors and exceptions with the following set of linkage errors:

1. NoSuchMethodError - thrown if an application tries to call a method, and this method does not exist. This usually indicates an incompatible change of the name or signature of the method. Many of the puzzlers, including most questions in the method descriptor category, cause this error to be thrown.

2. NoSuchFieldError - thrown if an application tries to access a field, and this field does not exist. This usually indicates an incompatible change of the name or the type of the field. The puzzlers in the auto (un)boxing category cause this error to be thrown.

3. InstantiationError - thrown when an application tries to instantiate an abstract class or interface using the new keyword.

4. IncompatibleClassChangeError - thrown when an incompatible class change has occurred to some class definition. This is the superclass of NoSuchMethodError, NoSuchFieldError and InstantiationError.

5. NoClassDefFoundError - thrown if an application tries to load a class, and this class cannot be found. This might be the result of changing the name of a class, or moving it into another package.

6. ClassFormatError - thrown when the JVM encounters a malformed class file. A common reason is that the version of the byte code is not supported by the JVM used.

We have used the Google search engine to assess the frequency of errors and exceptions reported. While this methods is not very precise, it is sufficient for us as we are mainly interested in the number of linkage errors relative to well-known programming errors and exceptions. We assume that both linkage errors and the errors and exceptions used for baselining equally suffer from false positives and false negatives.

We selected three popular open sources hosting sites according to [17]: GitHub, SourceForge and Google Code. We used the inurl attribute in Google queries to restrict the search to the issues tracking system of the respective project

hosting services. We also searched the popular Stackoverflow Q\&A site. The queries are listed in table 3, all queries were executed on 14 March 2014.

\subsection{Results}

Table 4 shows the number of pages with references to the respective errors and exceptions found. The ratios differ significantly between different hosting 
Table 3: Google queries used

\begin{tabular}{|l|l|}
\hline site & query \\
\hline \hline github & java.lang.NoSuchMethodError site:github.com \\
& inurl:issues \\
sourceforge & java.lang.NoSuchMethodError \\
& site:http://sourceforge.net/p inurl:bugs \\
google & java.lang.NoSuchMethodError \\
code & site:https://code.google.com/p inurl:issues \\
stackoverflowjava.lang.NoSuchMethodError site:stackoverflow.com
\end{tabular}

sites. This is sometimes caused by few very acive projects with a large number of issues. For instance, a large number of NoSuchFieldErrors appears in Google code issues. One of the Google code projects is google-web-toolkit. This project alone has 2,500 pages in its issue tracking system referencing NoSuchFieldError.

Overall, the number of linkage errors referenced is surprisingly high. In particular, NoSuchMethodError is in the same order of magnitude as NullpointerException, probably the most commonly encountered exception type in Java. In all issues tracking systems we investigated, NoSuchMethodError is more often referenced than both StackOverflowError and OutOfMemoryError. This indicates that compatibility problems already have a significant impact on the quality of products and the productivity of developers.

Table 4: Errors and exception reported

\begin{tabular}{|l|llll|}
\hline error or exception type & $\begin{array}{l}\text { github } \\
\text { issues }\end{array}$ & $\begin{array}{l}\text { google } \\
\text { code } \\
\text { issues }\end{array}$ & $\begin{array}{l}\text { source- } \\
\text { forge } \\
\text { bugs }\end{array}$ & $\begin{array}{l}\text { stack- } \\
\text { overflow }\end{array}$ \\
\hline \hline NullpointerException & 9,450 & 9,250 & 7,270 & 76,300 \\
ClassCastException & 2,030 & 6,450 & 993 & 19,600 \\
StackOverflowError & 499 & 3,630 & 237 & 51,500 \\
OutOfMemoryError & 1,080 & 4,790 & 426 & 18,000 \\
\hline NoSuchMethodError & 1,700 & 6,580 & 529 & 11,500 \\
NoSuchFieldError & 553 & 17,400 & 108 & 2,620 \\
InstantiationError & 12 & 45 & 1 & 786 \\
IncompatibleClassChangeErrb89 & 1,770 & 51 & 2,340 \\
NoClassDefFoundError & 3,240 & 2,910 & 976 & 54,700 \\
ClassFormatError & 58 & 346 & 71 & 1,600 \\
\hline
\end{tabular}




\section{Threats to Validity}

\subsection{Survey - Selection bias}

The aim of this study was to find out what programmers know about compatibility. Indeed, most of the respondents are programmers: 282 of 338 respondents who answered the background question $(83.43 \%)$. Note that only a very small number of respondents are students (30 of 338 respondents who answered the background question, $8.88 \%$ ). There is some uncertainty as 76 respondents $(18.35 \%)$ did not answer the background questions.

We cannot be sure how representative the population is, however, the size gives us some confidence. We recruited respondents by advertising the survey via the Java world ${ }^{5}$ portal and several Java user groups. Assuming that experienced developers are over-represented in these communities, the level of knowledge about compatibility among average programmers might actually be worse.

\subsection{Impact Analysis - Selection Bias}

We have only investigated projects hosted on three selected open-source hosting sites. However, we followed an independent rating [17] to select hosting sites based on number of projects hosted, and the availability of a public issue tracking system.

We have only investigated open source systems. It is likely that the same issues appear in closed source systems, but we do not have access to a sufficiently large number of such systems to investigate this questions. It is reasonable to assume that closed source systems suffer from integration problems at least the same level as open source programs, perhaps even more so as they often represent more complex end-user products, while many open source products are single-purpose programs or libraries not intended for use by end users, but used as building blocks in more complex programs.

\subsection{Impact Analysis - False Positives}

The use of fully qualified error names makes false positives unlikely. Some false positives could be caused by answers when respondents were confused with similarity of Java Linkage Errors and exceptions produced by Java Reflection API (such as NoSuchMethodException vs NoSuchMethodError). On the other hand, we believe that the Java Reflection API is used by experienced users who have strong Java knowledge and thus do not tend to do such basic mistakes.

There is a small change that linkage error class names are referenced in issues with other causes, for instance for comparison. While this is possible, it is probably very rare. Also, the other error and exception classes would suffer from this issue as well at a similar rate, so this will have no significant effect on relative numbers.

\footnotetext{
${ }^{5}$ http://www.javaworld.com/article/2074970/java-library-evolution-puzzlers.html
} 


\subsection{Impact Analysis - False Negatives}

Since the queries try to match exact strings, it is possible that we miss results when class names are not spelled correctly. We assume that only a few people would type in fully qualified class names when reporting issues, instead, copy and paste is used. Almost all issues we inspected manually included copies of stack traces. But even if there was a significant number of false negatives, this would equally affect the linkage errors and the exceptions and errors used for benchmarking. Therefore, this would have no significant effect on the relative numbers.

\section{Conclusion, Related and Future Work}

We have presented the results of a survey where we asked developers to solve puzzlers in order to test their knowledge on the different types of compatibility between programs and the libraries these programs use. We found that while developers have sound knowledge about the rules of source compatibility used by the compiler, even experienced developers lack knowledge about the rules of binary compatibility used by the JVM during linking.

We also demonstrated that errors which occur during linking are very common. This seems to be consistent with a trend away from building systems from scratch: more problems occur now at the boundaries between the actual program and code from libraries used by the program.

The question arises what can be done to improve the situations.

Firstly, better education of programmers is needed. There are several good resources available to the developer community, including some of the blog posts and presentations by Alex Buckley [6], Joseph D. Darcy [9, 10], Ian Robertson [24] and Jim des Rivieres [11]. We see this paper and the set of puzzlers as part of the effort.

Secondly, tools are needed to make linking smoother to facilitate library evolution. Some existing research has started to address this on different levels, including the generation of adapters to bridge "API gaps" in source code via refactoring $[7,2,16]$ and through byte code manipulation and instrumentation during class loading $[19,23,14]$. Another possible approach is to change the linker itself, i.e. to build a smarter JVM with linking rules more closely aligned to the rules of source compatibility. Such a linker could support specialising return types, and would enforce the catch or re-throw rule for checked exceptions. To the best of our knowledge, this has not yet been attempted.

Thirdly, better tools are needed to check assemblies (programs and the libraries they transitively depends on) for consistency. These tools could then be integrated into automated builds. An example is the Maven plugin that performs static byte-code verification proposed in [18] . Another existing tool that is fairly popular amongst developers is $\mathrm{Clirr}^{6}$. However, we found that

\footnotetext{
${ }^{6}$ http://sourceforge.net/projects/clirr/
} 
Clirr has multiple shortcomings with respect to compatibility problems related to declared exceptions and generic type parameters.

Finally, we notice that compatibility is complex, and that it is important to shield developers from this complexity to allow them to focus on the actual programming task. A popular method to address this is the use of semantic versioning schemes. In the Java technology space, such schemes are used in different technologies, including OSGi and Maven. Semantic versioning schemes implicitly promise contractual relationships between the providers and the consumers of APIs: if a consumer declares a requirement to use a library or module with a version within a certain range, then it is inferred that this consumer is compatible with any library or module that has a version number matching this range. In reality, this does not always work as version numbers are still assigned manually, sometimes influenced by non technical considerations such as marketing. What is therefore needed is standard tooling that can generate semantic versions complying with specifications such as the OSGi versioning policy [21] or the independent Semantic Versioning initiative [22]. Automated semantic versioning has been investigated by Bauml et al [3]. Bndtools [1] is a Java-based tool used by the OSGi community that supports semantic versioning.

\section{Acknowledgments}

We are grateful to all developers who completed the survey. We are particularly grateful to Jeff Friesen and Athen O'Shea from Java World, and Kon Soulianidis from the Melbourne Java user group. Without them, we would not have reached the critical number of responses. We would like to thank Alex Buckley and Hussain Al-Mutawa for their feedback on the puzzlers we used as questions in the survey.

The work was partially supported by European Regional Development Fund (ERDF), project "NTIS - New Technologies for the Information Society", European Centre of Excellence, CZ.1.05/1.1.00/02.0090.

\section{References}

[1] BND Tools. http://bndtools.org/. [Accessed: March 27, 2014], 2014.

[2] Ittai Balaban, Frank Tip, and Robert Fuhrer. Refactoring support for class library migration. In Proceedings OOPSLA '05, OOPSLA '05, pages 265-279, New York, NY, USA, 2005. ACM.

[3] Jaroslav Bauml and Premek Brada. Automated Versioning in OSGi: A Mechanism for Component Software Consistency Guarantee. In Proceedings SEAA '09, pages 428-435, 2009.

[4] Alex Blewitt. Jigsaw, Second Cut. http://www.infoq.com/news/2013/ 08/jigseen-it-before. [Accessed: March. 27, 2014], 2013. 
[5] Joshua Bloch and Neal Gafter. Java Puzzlers: Traps, Pitfalls, and Corner Cases. Addison-Wesley Professional, 2005.

[6] Alex Buckley. Different kinds of compatibility. https://blogs.oracle. com/abuckley/entry/on_compatibility. [Accessed: March 27, 2014], 2007.

[7] Kingsum Chow and David Notkin. Semi-automatic update of applications in response to library changes. In Proceedings ICSM'96, pages 359-. IEEE Computer Society, 1996.

[8] Bradley E Cossette and Robert J Walker. Seeking the ground truth: a retroactive study on the evolution and migration of software libraries. In Proceedings FSE'12, page 55. ACM, 2012.

[9] Joseph D. Darcy. Kinds of compatibility: Source, binary, and behavioral. https://blogs.oracle.com/darcy/entry/kinds_of_compatibility. [Accessed: March 27, 2014], 2008.

[10] Joseph D. Darcy. JDK release types and compatibility regions. https://blogs.oracle.com/darcy/entry/release_types_ compatibility_regions. [Accessed: March 27, 2014], 2009.

[11] Jim des Rivières. Evolving Java-based APIs. http://wiki.eclipse.org/ Evolving_Java-based_APIs. [Accessed: March. 27, 2014], 2007.

[12] Jens Dietrich, Kamil Jezek, and Premek Brada. Broken promises - an empirical study into evolution problems in java programs caused by library upgrades. In Proceedings CSRMR'14. IEEE, 2014.

[13] Danny Dig and Ralph Johnson. How do APIs evolve? A story of refactoring. Journal of Software Maintenance and Evolution: Research and Practice, 18(2):83-107, 2006.

[14] Danny Dig, Stas Negara, Vibhu Mohindra, and Ralph Johnson. ReBA: refactoring-aware binary adaptation of evolving libraries. In Proceedings ICSE '08, pages 441-450, New York, NY, USA, 2008. ACM.

[15] James Gosling, Bill Joy, Guy Steele, Gilad Bracha, and Alex Buckley. The Java ${ }^{T M}$ Language Specification 7 th Edition. Oracle, Inc., California, USA, 2012 .

[16] J. Henkel and A. Diwan. Catchup! capturing and replaying refactorings to support api evolution. In Proceedings ICSE'2005, pages 274-283, May 2005 .

[17] Neil Chue Hong. Choosing a repository for your software project. http://software.ac.uk/resources/guides/ choosing-repository-your-software-project, 2013. 
[18] Kamil Jezek, Lukas Holy, Antonin Slezacek, and Premek Brada. Software components compatibility verification based on static byte-code analysis. In SEAA, 39th EUROMICRO, pages 145 - 152, 2013.

[19] Ralph Keller and Urs Hölzle. Binary Component Adaptation. In Proceedings ECOOP '98, volume 1445, pages 307-329. Springer, 1998.

[20] Tim Lindholm, Frank Yellin, Gilad Bracha, and Alex Buckley. The Java ${ }^{T M}$ Virtual Machine Specification - Java ${ }^{T M}$ SE 7 Edition. Oracle, Inc., 2012 .

[21] OSGi Alliance. Semantic Versioning Technical Whitepaper Revision 1.0. http://www.osgi.org/wiki/uploads/Links/SemanticVersioning. pdf. [Accessed: March 27, 2014], 2010.

[22] Tom Preston-Werner. Semantic Versioning 2.0.0. http://semver.org/. [Accessed: March 27, 2014], 2010.

[23] Mario Pukall, Alexander Grebhahn, Reimar Schröter, Christian Kästner, Walter Cazzola, and Sebastian Götz. Javadaptor: Unrestricted dynamic software updates for java. In Proceedings ICSE'11, ICSE '11, pages 989991, New York, NY, USA, 2011. ACM.

[24] Ian Robertson. The science and art of backwards compatibility. JavaOne 2013, http://www.slideshare.net/nainostrebor/ the-science-and-art-of-backwards-compatibility. [Accessed: March 27, 2014], 2013.

[25] The OSGi Alliance. OSGi core release 5. http://www.osgi.org/ Specifications, 2012. 